hep-th/0610113

\title{
Elliptic Flow, Kasner Universe and Holographic Dual of RHIC Fireball
}

\author{
Sang-Jin Sin ${ }^{a, b *}$, Shin Nakamura ${ }^{a, c} \dagger$ and Sang Pyo Kim ${ }^{b, d} \ddagger$ \\ ${ }^{a}$ BK21 Program Division in Physics, Department of physics, \\ Hanyang University, Seoul 133-791, Korea \\ ${ }^{b}$ Asia Pacific Center for Theoretical Physics, Postech, Pohang 790-784, Korea \\ ${ }^{c}$ Center for Quantum Spacetime, Sogang University, Seoul 121-742, Korea \\ ${ }^{d}$ Department of Physics, Kunsan National University, Kunsan 573-701, Korea
}

October 10, 2006

\begin{abstract}
We consider a holographic dual of hydrodynamics of $\mathcal{N}=4 \mathrm{SYM}$ plasma that undergoes non-isotropic three-dimensional expansion relevant to RHIC fireball. Our model is a natural extension of the Bjorken's one-dimensional expansion, and it describes an elliptic flow whose $v_{2}$ and eccentricity are given in terms of anisotropy parameters. Holographic renormalization shows that absence of conformal anomaly in the SYM theory constrains our local rest frame to be a Kasner spacetime. We show that the Kasner spcetime provides a simple description of the anisotropically expanding fluid within a well-controled approximation. We also find that the dual geometry determines some of the hydrodynamic quantities in terms of the initial condition and the fundamental constants.
\end{abstract}

\footnotetext{
${ }^{*}$ E-mail: sjsin@hanyang.ac.kr

${ }^{\dagger}$ E-mail: nakamura@hanyang.ac.kr

${ }^{\ddagger}$ E-mail: sangkim@kunsan.ac.kr
} 


\section{Introduction}

There has been much hope that one might be able to use AdS/CFT [1] to describe the real systems after certain amount of deformations. In fact it has been suggested that the fireball in Relativistic Heavy Ion Collision (RHIC) can be explained from dual gravity point of view [2, 3, 4, 5], since the quark-gluon plasma (QGP) created there are in the strong coupling region [6, 7]. Although the YM theory described by the standard AdS/CFT is large- $N_{c} \mathcal{N}=4$ SYM theory, there are many attempts to construct models closer to QCD [8]. SUSY is not very relevant in the finite temperature context because it is broken completely.

Since the RHIC fireball is expanding, we need to understand AdS/CFT in time dependent situations. Recently, Janik and Peschanski [9, 10] discussed this problem in non-viscous cases. They use the conservation law and conformal invariance together with the holographic renormalization [11, 12] to express the bulk geometry from the given boundary data. As a result, the bulk geometry reproduces the basic features of Bjorken theory [13]. Theses results were generalized to the cases where shear viscosity is included [14]. Indeed, it had been pointed out that inclusion of shear viscosity is very important in the analysis of real RHIC physics since it plays an essential role in the elliptic flow (see for example, [15, 16, 17, 18, 19, 20]). The shear viscosity at the strong coupling limit was calculated for the $\mathcal{N}=4 \mathrm{SYM}$ systems in 21] using AdS/CFT, which fits the perfect fluidity of RHIC QGP (see [7, 16, 22] and the references therein).

In this paper, we consider a holographic dual of strongly interacting $\mathcal{N}=4$ large- $N_{c}$ SYM fluid with non-isotropic three-dimensional expansion which is relevant to "Little Bang" of RHIC. We will first make a simplest generalization of the Bjorken's one-dimensional expansion to three-dimensional cases. The resulting local rest frame of the fluid is described by the Kasner metric whose extreme limit reproduces the Bjorken hydrodynamics. Interestingly, the hydrodynamic equation and the equation of state will be shown to be independent of the non-isotropy parameters.

Further more, using holographic renormalization, we will establish the holographic dual geometry of the anisotropically expanding fluid in the Kasner spacetime and discuss its physical consequences by extending the work of [9, 14]. Interestingly, the gravity dual carries more information than the hydrodynamics which is our input. The integration constants coming from the hydrodynamic equation cannot be determined by the hydrodynamics itself. However, we will show that such quantities can be determined by considering the dual geometry. Therefore it provides a method to extract useful information on the macroscopic properties of the expanding fluid in terms of microscopic data.

Kasner spacetime is a curved spacetime in general. However, we find that the Kasner spacetime can be a well-controled approximation of a local rest frame of an anisotropically expanding fluid on Minkowski spacetime. Therefore, the Kasner spacetime provides a useful framework for analysing the three-dimensional expansion because of the simplification of the hydrodynamic equations.

The organization of the paper is the following. In Section 2, we introduce Kasner spacetime as a local rest frame of a fluid under three-dimensional anisotropic expansion. We also establish the 
hydrodynamics based on that local rest frame. Section 3 gives analyses in the gravity dual in the late time regime. We extend the results of [9, 14] to the case of our interest: the three-dimensional anisotropic expansion with shear viscosity. We show that the dual geometry determines some of the hydrodynamic quantities in terms of the initial condition and the fundamental constants. In Section 4 we show that the hydrodynamics on the Kasner spacetime (and hence its gravity dual) describes an elliptic flow on the flat spacetime within a well-controlled approximation. We analyze the properties of the flow. We conclude in the final section. The definition of the approximation and its justification are given in appendix.

\section{Hydrodynamics in Kasner space and anisotropic expansion}

The remarkable success of Bjorken's hydrodynamics needs to be extended for more realistic cases of three-dimensional expansions. The Bjorken's basic assumption is existence of the so-called "central rapidity region" (CPR). The local rest frame of the fluid can be given by $\operatorname{propertime}(\tau)$-rapidity $(y)$, whose relationship with the cartesian coordinate is $\left(X^{0}, X^{1}, X^{2}, X^{3}\right)=\left(\tau \cosh y, \tau \sinh y, X^{2}, X^{3}\right)$.

We have chosen the collision axis to be in the $x^{1}$ direction. The Minkowski metric in this coordinate has the form

$$
d s^{2}=-d \tau^{2}+\tau^{2} d y^{2}+\left(d X^{2}\right)^{2}+\left(d X^{3}\right)^{2}
$$

Our starting point is an observation that the above metric describes a one-dimensional Hubble expansion, i.e., an expansion of the universe instead of that of the fluid, in which the rapidity plays the role of the co-moving coordinate. The hydrodynamic equations can be derived from the covariant conservation of the energy-momentum tensor with the above metric.

The real expansion in RHIC is not one-dimensional but a three-dimensional one, although the dominant flow is along the collision axis. The idea is that since the one-dimensional approximation of RHIC fireball expansion is seen as a Hubble flow, a three-dimensional Hubble flow may describe the RHIC plasma better. Let us begin with an ansatz for a local rest frame given by

$$
d s^{2}=-d \tau^{2}+\tau^{2 a}\left(d x^{1}\right)^{2}+\tau^{2 b}\left(d x^{2}\right)^{2}+\tau^{2 c}\left(d x^{3}\right)^{2} .
$$

Here $x^{i}, i=1,2,3$ denote the co-moving coordinates. The $a, b$ and $c$ are arbitrary constants for a moment. However, as we will show in Section 3, they satisfy

$$
a+b+c=1, \quad a^{2}+b^{2}+c^{2}=1,
$$

if we impose conformal invariance of the fluid. Under the above conditions, the metric is called Kasner metric describing a homogeneous but anisotropic expansion of the Universe. In short, we identify the "Little Bang" in "SYM version of RHIC" with a Big Bang with the homogeneous but anisotropic expansion described by the Kasner metric. We use this metric in the late time regime because we describe only the late time evolution of the fluid where hydrodynamics is valid. Therefore 
the initial singularity of the metric is not a relevant feature for us. Since we take $x^{1}$ as the longitudinal direction, a realistic set up is to choose $a \sim 1$ and $b, c \sim 0$ (see section 4).

We first establish the hydrodynamics in the Kasner metric 1 We assume that the expansion is anisotropic but homogeneous, and the physical quantities depend only on $\tau$. Using the fact that the energy-momentum tensor is diagonal on the local rest frame, and using the symmetry in transverse coordinates, we can write

$$
T^{\mu}{ }_{\nu}=\operatorname{diag}\left(-\rho, f_{1}, f_{2}, f_{3}\right),
$$

where $\rho$ is the energy density of the fluid. By use of the conservation law $\nabla_{\mu} T^{\mu \nu}=0$ we get

$$
\dot{\rho}+\rho / \tau+\sum_{i} a_{i} f_{i} / \tau=0,
$$

and from the conformal invariance $T_{\mu}^{\mu}=0$, we get

$$
-\rho+\sum f_{i}=0
$$

To get the above results, we have used the following non-zero $\Gamma_{\alpha \beta}^{\mu}$ 's:

$$
\Gamma_{11}^{\tau}=a \tau^{2 a-1}, \quad \Gamma_{22}^{\tau}=b \tau^{2 b-1}, \quad \Gamma_{33}^{\tau}=c \tau^{2 c-1}, \quad \Gamma_{1 \tau}^{1}=a / \tau, \quad \Gamma_{2 \tau}^{2}=b / \tau, \quad \Gamma_{3 \tau}^{1}=c / \tau .
$$

On the other hand, the energy-momentum tensor in the framework of relativistic hydrodynamics is known to be

$$
T^{\mu \nu}=(\rho+P) u^{\mu} u^{\nu}+P g^{\mu \nu}+\tau^{\mu \nu},
$$

where $P$ is the pressure of the fluid, $u^{\mu}=(\gamma, \gamma \vec{v})$ is the four-velocity field in terms of the local fluid velocity $\vec{v}$, and $\tau^{\mu \nu}$ is the dissipative term. In a frame where the energy three-flux vanishes, $\tau^{\mu \nu}$ is given in terms of the bulk viscosity $\xi$ and the shear viscosity $\eta$ by

$$
\tau^{\mu \nu}=-\eta\left(\triangle^{\mu \lambda} \nabla_{\lambda} u^{\nu}+\triangle^{\nu \lambda} \nabla_{\lambda} u^{\mu}-\frac{2}{3} \triangle^{\mu \nu} \nabla_{\lambda} u^{\lambda}\right)-\xi \triangle^{\mu \nu} \nabla_{\lambda} u^{\lambda}
$$

under the assumption that $\tau^{\mu \nu}$ is of first order in gradients. We have defined the three-frame projection tensor as $\triangle^{\mu \nu}=g^{\mu \nu}+u^{\mu} u^{\nu}$. For the conformal invariance, we set $\xi=0$. Notice that the bulk viscosity in the realistic RHIC setup is also negligible. (See for example, Ref. [16].)

The four-velocity of the fluid at any point in the local rest frame is $u^{\mu}=(1,0,0,0)$, and this makes the energy-momentum tensor diagonal. Using $\Delta^{\mu \nu}=\operatorname{diag}\left(0, \tau^{-2 a}, \tau^{-2 b}, \tau^{-2 c}\right), \nabla_{\lambda} u^{\nu}=\Gamma_{\lambda 0}^{\nu}=$ $\operatorname{diag}(0, a / \tau, b / \tau, c / \tau)$ and $\nabla_{\nu} u^{\nu}=(a+b+c) / \tau$, we get the mixed energy-momentum tensor:

$$
T^{\mu}{ }_{\nu}=\left(\begin{array}{cccc}
-\rho & 0 & 0 & 0 \\
0 & P-\frac{2}{3}(3 a-1) \frac{\eta}{\tau} & 0 & 0 \\
0 & 0 & P-\frac{2}{3}(3 b-1) \frac{\eta}{\tau} & 0 \\
0 & 0 & 0 & P-\frac{2}{3}(3 c-1) \frac{\eta}{\tau}
\end{array}\right) .
$$

\footnotetext{
${ }^{1}$ We use the late time approximation, which is employed in [14], where the macroscopic quantities are assumed to evolve sufficiently slowly.
} 
By identifying (2.10) with (2.4), we obtain

$$
f_{i}=p-\left(a_{i}-\frac{1}{3}\right) \frac{2 \eta}{\tau}
$$

where $a_{i}=a, b, c$ for $i=1,2,3$ respectively. Inserting these into (2.5) and (2.6), we get

$$
\dot{\rho}+\frac{1}{\tau} \rho+\sum_{i} \frac{a_{i}}{\tau}\left(p-\left(a_{i}-\frac{1}{3}\right) \frac{2 \eta}{\tau}\right)=0
$$

and

$$
-\rho+3 p-2 \eta\left(\frac{\sum_{i} a_{i}-1}{\tau}\right)=0
$$

Using the second equation, we may write the first equation in terms of the energy density as

$$
\dot{\rho}+\left(1+\frac{1}{3} \sum_{i} a_{i}\right) \frac{\rho}{\tau}=\left(\sum_{i} a_{i}^{2}-\frac{1}{3}\left(\sum_{i} a_{i}\right)^{2}\right) \frac{2 \eta}{\tau^{2}} .
$$

As we will discuss in Section 3, if we impose the conformal invariance, we get the conditions

$$
\sum_{i} a_{i}=1, \quad \sum_{i} a_{i}^{2}=1
$$

under which the equation of state and the conservation law become:

$$
\begin{aligned}
p & =\frac{\rho}{3} \\
\frac{d \rho}{d \tau}+\frac{4}{3} \frac{\rho}{\tau} & =\frac{4}{3} \frac{\eta}{\tau^{2}} .
\end{aligned}
$$

Remarkably, the equations for fluid dynamics are completely independent of the parameters $a, b, c$ in this case. In fact the dynamical law should not depend on the initial conditions $(a, b$ and $c)$ that reflects the initial collision geometry. In this respect, (2.15) leads us a satisfactory consequence.

Notice that both of $\rho$ and $\eta$ depend on the proper time $\tau$ in general. Let's assume that the shear viscosity evolves by $\eta=\eta_{0} / \tau^{\beta}$, where $\eta_{0}$ is a positive constant. The solution of (2.16) is then given by

$$
\begin{aligned}
\rho(\tau) & =\frac{\rho_{0}}{\tau^{4 / 3}}+\frac{4 / 3}{1 / 3-\beta} \frac{\eta_{0}}{\tau^{1+\beta}} \quad(\text { for } \beta \neq 1 / 3), \\
& =\frac{\rho_{0}}{\tau^{4 / 3}}+\frac{4 \eta_{0}}{3} \frac{\ln \tau}{\tau^{4 / 3}} \quad(\text { for } \beta=1 / 3),
\end{aligned}
$$

where $\rho_{0}$ is a positive constant. For $\beta \leq 1 / 3$ case, the viscous corrections in the hydrodynamic quantities become dominant in the late time, which invalidates the hydrodynamic description. If $\beta>1 / 3$, the shear viscosity term is subleading in the late time as we expect. Therefore we will consider only the latter case from now on.

The proper-time dependence of the temperature $T$ can be read off by assuming the StefanBoltzmann's law $\rho \propto T^{4}$ :

$$
T=T_{0}\left(\frac{1}{\tau^{1 / 3}}+\frac{1 / 3}{1 / 3-\beta} \frac{\eta_{0}}{\rho_{0}} \frac{1}{\tau^{\beta}}+\cdots\right)
$$


In the static finite temperature system of strongly coupled $\mathcal{N}=4 \mathrm{SYM}$ theory, it is known that $\eta \propto T^{3}[21$. Let us assume that the result is valid in the slowly varying non-static cases. Then we can set $\beta=1$ hence

$$
\eta=\frac{\eta_{0}}{\tau}
$$

We know $\rho \sim T^{4}$ and $\eta \sim T^{3}$ can be consistent only if there is an additional term in (2.19), but the correction term is negligible in our case. The temperature behavior is then given by

$$
T=T_{0}\left(\frac{1}{\tau^{1 / 3}}-\frac{1}{2} \frac{\eta_{0}}{\rho_{0}} \frac{1}{\tau}+\cdots\right) .
$$

We can evaluate the entropy change in the presence of shear viscosity by using hydrodynamics. The conservation of energy-momentum tensor can be rewritten as

$$
\frac{d(\sqrt{g} \rho)}{d \tau}+\frac{d \sqrt{g}}{d \tau} P=\frac{4}{3} \frac{\sqrt{g} \eta}{\tau^{2}}
$$

where $\sqrt{g}=\tau$ is the volume element in the co-moving coordinate. By integrating over the unit volume in the co-moving coordinate, and using the thermodynamic relation between the entropy and energy-work,

$$
d E+P d V=T d S
$$

(2.21) can be written as

$$
T \frac{d(\sqrt{g} s)}{d \tau}=\frac{4}{3} \frac{\eta \sqrt{g}}{\tau^{2}}
$$

where $s$ denotes the entropy density and $\sqrt{g} s \equiv S$ is the entropy per unit co-moving volume. Notice that in the absence of viscosity, the entropy per unit co-moving volume is constant. Now, the entropy per unit co-moving volume has the time dependence:

$$
\begin{aligned}
S(\tau) & =S_{0}+\frac{4}{3} \int_{0}^{\tau} d \tau \frac{\eta \sqrt{g}}{\tau^{2} T} \\
& =S_{\infty}-2 \frac{\eta_{0}}{T_{0}} \tau^{-2 / 3}+\cdots,
\end{aligned}
$$

where $S_{\infty}=S(\infty)$. The dissipation creates entropy but its rate slows down with time. Notice that all these arguments are completely in parallel with the case of the one-dimensional expansion.

The hydrodynamics does not calculate the constants $S_{\infty}$ and $T_{0}$ in terms of the initial condition. It is important to point out that by embedding the hydrodynamics into AdS/CFT, we can determine these parameters in terms of the initial condition $\rho_{0}$ and the fundamental constants of the theory.

\section{Holographic dual of anisotropic expansion}

In this section, we will find a five-dimensional metric that is dual to the hydrodynamic description of the YM fluid in the previous section. Some of the parameters of hydrodynamics will be determined 
as a consequence. The basic strategy is to use the Einstein's equation together with the boundary condition given by the four-dimensional energy-momentum tensor [11, 12, 9]. We consider general asymptotically AdS metrics in the Fefferman-Graham coordinate:

$$
d s^{2}=r_{0}^{2} \frac{g_{\mu \nu} d x^{\mu} d x^{\nu}+d z^{2}}{z^{2}}
$$

where $x^{\mu}=\left(\tau, x^{1}, x^{2}, x^{3}\right)$ in our case. $r_{0} \equiv\left(4 \pi g_{s} N_{c} \alpha^{2}\right)^{1 / 4}$ is the length scale given by the string coupling $g_{s}$ and the number of the colors $N_{c}$. The four-dimensional metric $g_{\mu \nu}$ is expanded with respect to $z$ in the following form [11, 12]:

$$
g_{\mu \nu}(\tau, z)=g_{\mu \nu}^{(0)}(\tau)+z^{2} g_{\mu \nu}^{(2)}(\tau)+z^{4} g_{\mu \nu}^{(4)}(\tau)+z^{6} g_{\mu \nu}^{(6)}(\tau)+\cdots
$$

Here $g_{\mu \nu}^{(0)}$ is the physical four-dimensional metric for the gauge theory on the boundary, which is given by (2.2) in the present case. The $g_{\mu \nu}^{(n)}$ 's depend only on $\tau$ because our physical quantities are assumed to depend only on $\tau$.

$g_{\mu \nu}^{(2)}$ is related to the conformal anomaly of the YM theory in the following way [1]:

$$
\left\langle T_{\mu}^{\mu}\right\rangle=-\frac{1}{16 \pi G_{5}}\left[\left(\operatorname{Tr} g^{(2)}\right)^{2}-\operatorname{Tr}\left(g^{(2)}\right)^{2}\right]
$$

where $G_{5}$ is the 5 d Newton's constant given by $G_{5}=8 \pi^{3} \alpha^{\prime 4} g_{s}^{2} / r_{0}^{5}$ so that

$$
\frac{4 \pi G_{5}}{r_{0}^{3}}=\frac{2 \pi^{2}}{N_{c}^{2}}
$$

in our notation. Since we are dealing with $\mathcal{N}=4 \mathrm{SYM}$ theory on $R^{1,3}$, we should require the conformal invariance: $\left\langle T_{\mu}^{\mu}\right\rangle=0$. The most natural choice is given by

$$
g_{\mu \nu}^{(2)}=0
$$

This is equivalent to the Ricci flat condition for the four-dimensional metric:

$$
R_{\mu \nu}=0
$$

through the relationship [1]

$$
g_{\mu \nu}^{(2)}=\frac{1}{2}\left(R_{\mu \nu}-\frac{1}{6} R g_{\mu \nu}^{(0)}\right)
$$

Since

$$
R_{00}=\left(\sum_{i} a_{i}-\sum_{i} a_{i}^{2}\right) / \tau^{2}, \quad R_{i i}=a_{i}\left(\sum_{j} a_{j}-1\right) \tau^{2 a_{i}-2},
$$

the Ricci flat condition (3.6) gives the Kasner condition (2.3).

One should notice that we are not solving the four-dimensional Einstein's equation in the presence of $T_{\mu \nu}$. The Kasner metric is not a consequence of the gravitational effect of $T_{\mu \nu}$, but an effective 
description of the spacetime expansion satisfying the conformal invariance of strong gauge theory interaction.

We can identify the first non-trivial data in (3.2),$g_{\mu \nu}^{(4)}$, with the energy-momentum tensor at the boundary [11]:

$$
g_{\mu \nu}^{(4)}=\frac{4 \pi G_{5}}{r_{0}^{3}}\left\langle T_{\mu \nu}\right\rangle
$$

in our notation. For the time being, we set $4 \pi G_{5}=1$ and $r_{0}=1$. The higher-order terms in (3.2) are determined by solving the Einstein's equation with the negative cosmological constant $\Lambda=-6$ [11] (see also [9]):

$$
R_{M N}-\frac{1}{2} G_{M N} R-6 G_{M N}=0,
$$

where the metric and the curvature tensor are the five-dimensional ones of (3.1). $g_{\mu \nu}^{(2 n)}$ is described by $g_{\mu \nu}^{(2 n-2)}, g_{\mu \nu}^{(2 n-4)}, \cdots, g_{\mu \nu}^{(0)}$ through the Einstein's equation in five dimensions. In other words, we can obtain the higher-order terms in (3.2) recursively by starting with the initial data $g_{\mu \nu}^{(0)}(\sim$ Kasner) and $g_{\mu \nu}^{(4)}\left(\sim T_{\mu \nu}\right)$.

Let us come back to our main interest to obtain the bulk geometry in the presence of shear viscosity. The energy-momentum tensor for $\beta=1$ is written by using (2.17) as

$$
T_{\nu}^{\mu}=\left(\begin{array}{cccc}
-\frac{\rho_{0}}{\tau^{4 / 3}}+\frac{2 \eta_{0}}{\tau^{2}} & 0 & 0 & 0 \\
0 & \frac{\rho_{0}}{3 \tau^{4 / 3}}-\frac{2 a \eta_{0}}{\tau^{2}} & 0 & 0 \\
0 & 0 & \frac{\rho_{0}}{3 \tau^{4 / 3}}-\frac{2 b \eta_{0}}{\tau^{2}} & 0 \\
0 & 0 & 0 & \frac{\rho_{0}}{3 \tau^{4 / 3}}-\frac{2 c \eta_{0}}{\tau^{2}}
\end{array}\right)
$$

We find that the metric components, $g_{\tau \tau}, g_{y y} / \tau^{2}, g_{x x}$ have the following structure by solving the Einstein's equation recursively:

$$
f^{(1)}(v)+\eta_{0} h^{(1)}(v) / \tau^{2 / 3}+\tilde{f}^{(2)}(v) / \tau^{4 / 3}+\cdots,
$$

Note that the viscosity dependent terms exist at the order of $\tau^{-2 / 3}$ and these are more important than the higher-order terms neglected in [9]. We are considering the late time region $\tau \gg 1$. But to see the effects of the viscosity, we need to keep the terms at least to the order of $\tau^{-2 / 3}$. In this paper we consider the viscosity effects to the minimal order.

Now we solve the Einstein's equation recursively. The power series that appear in the solution can be re-summed to give a compact form of the metric. After some hard work, we can get the late time $5 \mathrm{~d}$ bulk geometry given by 2

$$
d s^{2}=\frac{1}{z^{2}}\left\{-\frac{\left(1-\frac{\rho z^{4}}{3}\right)^{2}}{1+\frac{\rho z^{4}}{3}} d \tau^{2}+\left(1+\frac{\rho z^{4}}{3}\right) \sum_{i=1}^{3}\left(\frac{1+\frac{\rho z^{4}}{3}}{1-\frac{\rho z^{4}}{3}}\right)^{\left(1-3 a_{i}\right) \gamma} \tau^{2 a_{i}}\left(d x^{i}\right)^{2}\right\}+\frac{d z^{2}}{z^{2}},
$$

\footnotetext{
${ }^{2}$ One should keep in mind that we are looking for the late time geometry; the metric (3.13) is correct only to the order of $\gamma$ and the $O\left(\gamma^{2}\right)$ contributions are not unambiguously determined. The representation of (3.13) is chosen since it makes the volume of the horizon finite.
} 
where

$$
\gamma \equiv \frac{\eta_{0}}{\rho_{0} \tau^{2 / 3}} \text { and } \rho=\frac{\rho_{0}}{\tau^{4 / 3}}-\frac{2 \eta_{0}}{\tau^{2}}
$$

Notice that the energy-momentum tensor (3.11) can NOT be written in terms of the whole $\rho(\tau)$. It is an amusing surprise to see that the final metric nevertheless can be written in terms of $\rho(\tau)$ (apart from the powers) in a compact form. This implies that the position of the horizon can be determined solely by the energy density.

\subsection{Macroscopic Parameters from Gravity Dual}

The Hawking temperature in the adiabatic approximation is given by

$$
T(\tau)=\sqrt{2} /\left(\pi z_{0}(\tau)\right)
$$

where

$$
z_{0}(\tau)=[3 / \rho(\tau)]^{1 / 4}
$$

is the time-dependent position of the horizon. Using these, we obtain

$$
\rho=\frac{3}{8} \pi^{2} N_{c}^{2} T^{4}(\tau)
$$

by restoring the normalization of $\rho: \rho \rightarrow 4 \pi G_{5} / r_{0}^{3} \cdot \rho$. The entropy per unit co-moving volume is given by

$$
\begin{aligned}
S & =\lim _{z \rightarrow z_{0}(\tau)} \frac{1}{4 G_{5}} \sqrt{\prod_{i=1}^{3}\left(1+\frac{\rho z^{4}}{3}\right)\left(\frac{1+\frac{\rho z^{4}}{3}}{1-\frac{\rho z^{4}}{3}}\right)^{\left(1-3 a_{i}\right) \gamma} \tau^{2 a_{i}}} \\
& =\frac{1}{4 G_{5}} \frac{2 \sqrt{2} \tau r_{0}^{3}}{z_{0}^{3}(\tau)} \\
& =N_{c}^{2} \frac{\sqrt{2}}{\pi}\left(\frac{2 \pi^{2}}{N_{c}^{2}} \frac{\rho_{0}}{3}\right)^{3 / 4}\left(1-\frac{3}{2} \frac{\eta_{0}}{\rho_{0} \tau^{2 / 3}}+O\left(\tau^{-4 / 3}\right)\right)
\end{aligned}
$$

Notice that the entropy is completely independent of geometric parameters (i.e., $\left.a_{i}\right)$. Without conformal invariance this result is not guaranteed. As we emphasized in [14], the value of $S$ at $\tau=\infty$, which cannot be determined by hydrodynamics alone, is precisely determined to be

$$
S_{\infty}=N_{c}^{2} \frac{\sqrt{2}}{\pi}\left(\frac{2 \pi^{2}}{N_{c}^{2}} \frac{\rho_{0}}{3}\right)^{3 / 4}
$$

in terms of the initial condition $\rho_{0}$ and the microscopic gauge theory parameter $N_{c}$. Similarly,

$$
T(\tau)=\frac{T_{0}}{\tau^{1 / 3}}(1-2 \gamma(\tau))^{1 / 4}, \quad \text { with } \quad T_{0}=\frac{\sqrt{2}}{\pi}\left(\frac{2 \pi^{2}}{N_{c}^{2}} \frac{\rho_{0}}{3}\right)^{1 / 4}
$$


These parameters $S_{\infty}, T_{0}$ are precisely the quantities used in macroscopic theory (hydrodynamics) which should be provided by a microscopic theory like QCD. What we are showing here is that by considering the AdS/CFT dual of hydrodynamics, one can determine such quantities.

Let us check consistency of (3.18) and (2.24). Apparent time dependence agrees in the leading order. In fact one can do more. The normalized entropy-creation rate from the gravity dual result (3.18) is given by

$$
\frac{1}{S} \frac{d S}{d \tau}=\frac{\eta_{0}}{\rho_{0} \tau^{5 / 3}}+O\left(\tau^{-7 / 3}\right)
$$

and that from hydrodynamics result (2.24) is

$$
\frac{1}{S} \frac{d S}{d \tau}=\frac{4}{3} \frac{\eta_{0}}{T_{0} S_{\infty} \tau^{5 / 3}}+O\left(\tau^{-7 / 3}\right) .
$$

Comparing (3.21) and (3.22), we obtain

$$
S_{\infty}=\frac{4}{3} \frac{\rho_{0}}{T_{0}}=\left.\frac{4}{3} \frac{\rho \tau}{T}\right|_{\tau=\infty}
$$

This is the consistency condition that is required to be checked 3 With use of $S_{\infty}$ and $T_{0}$ given in eqn's (3.19) and (3.20) we can check that the consistency condition (3.23) is indeed satisfied.

\section{Flow of RHIC fireball and Kasner spacetime}

So far, we have established the gravity dual of the Yang-Mills system in Kasner spacetime. Now we would like to suggest a relevance of our model to description of the elliptic flow in RHIC experiments. We have seen in section 2 that the hydrodynamic description of the three-dimensional expansion in the Kasner spacetime is as simple as that of the Bjorken expansion in the flat spacetime. Therefore, it is great if we can apply such a simple formalism to anisotropically expanding RHIC fireball. However, from the realistic point of view, we sacrificed the flatness of $4 \mathrm{~d}$ spacetime for the simplicity of the fluid dynamics of 3 dimensional expansion. One immediate question is when and under which condition we can justify it. The hydrodynamics on the Kasner spacetime provides a well-approximated description of a three-dimensional expansion in the flat spacetime if curvature which is small enough. Notice also that the spacetime symmetry crucial to our problem, which is uniformity of the spacetime, is maintained in Kasner spacetime.

In figure 1, we show the allowed region of the anisotropic parameters. $(b, c)=(0,0)$ corresponds to the Bjorken expansion. The non-zero components of the Riemann tensor of the Kasner spacetime are

$$
R_{0 i 0 i}=\left(1-a_{i}\right) a_{i} \tau^{2 a_{i}-2}, \quad R_{i j i j}=a_{i} a_{j} \tau^{2 a_{i}+2 a_{j}-2},
$$

\footnotetext{
${ }^{3}$ In fact this is the relationship among the entropy, the energy (per unit co-moving volume) and the temperature obtained by thermodynamics at $\tau=\infty$ where the system reaches thermal equilibrium.
} 


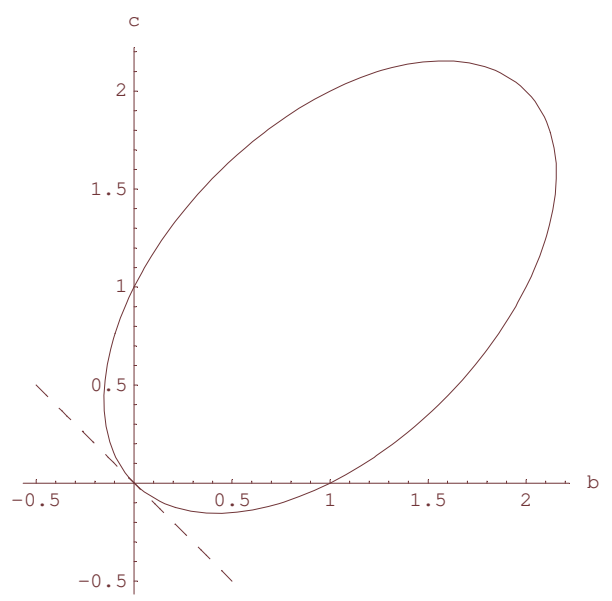

Figure 1: The available region of $(b, c)$ is on the ellipse between $(0,0)$ and $(1,0) .(b, c)=(0,0)$ is Bjorken point

and the non-flatness is directly related to the distance from the Bjorken point $(a, b, c)=(1,0,0)$ on the parameter space. In order to keep the deviation from the flat spacetime small, we restrict ourselves within the vicinity of the Bjorken point,

$$
a \simeq 1, \quad b \simeq 0, \quad c \simeq 0
$$

that corresponds to almost central collisions in RHIC.

In appendix, we show what approximation is necessary to reach kasner space starting from a flat spacetime. The conditions are: 1) the fluid is produced by almost central collision (small $b$ ), 2) we consider only the central part of the fluid (small $x^{\perp}$ ), and 3) we consider only the late time regime. For more detail, see appendix $\mathrm{A}$.

With these limitations in mind, let us consider how the elliptic flow can be described within our framework. We can choose $b \geq c$ without any loss of generality. By considering the intersection of the plane and the unit sphere in the $a, b, c$ space of (2.3), we can see that

$$
a>b>-c>0 \text {. }
$$

This means that one of the transverse directions must contract and the others expand so that the expansion is elliptical whose eccentricity grows and eventually saturate to 1. 4

The transverse expansion has a natural interpretation as the elliptic flow, one of the most concrete evidence for the strong nature of the interaction. Let $\varepsilon$ be the eccentricity defined by 5

$$
\varepsilon=\frac{\left\langle X_{2}^{2}-X_{3}^{2}\right\rangle_{X}}{\left\langle X_{2}^{2}+X_{3}^{2}\right\rangle_{X}}
$$

\footnotetext{
${ }^{4}$ There is no contraction in the realistic RHIC QGP. The contraction in the present model is due to the conformal invariance which is unavoidable for $\mathcal{N}=4 \mathrm{SYM}$ theory. Notice that this volume-preserving nature indicates our $\mathcal{N}=4$ SYM plasma is more "liquid-like" than the RHIC QGP.

${ }^{5}$ The $\varepsilon$ defined here contains an extra minus sign comparing to other literature such as [15, 16]. We define its positivity by the direction of the $v_{2}$ evolution.
} 
where $X^{i}$ is the cartesian coordinate and $\langle\cdots\rangle_{X} \equiv \int \cdots \rho d X_{2} d X_{3}$. On the other hand, $v_{2}$, the quantity experimentally characterizing the elliptic flow, is defined by (see for example, [15, 16, 20])

$$
\frac{1}{N} \frac{d N}{d \phi}=\frac{1}{2 \pi}\left(v_{0}+2 v_{2} \cos (2 \phi)+2 v_{4} \cos (4 \phi)+\cdots\right)
$$

where $N$ is the number of the partons and $\phi$ is the angular coordinate on the transverse momentum plane. It can be calculated from the following identification,

$$
v_{2}=\frac{\int d^{2} P_{T}\left(\frac{P_{2}^{2}-P_{3}^{2}}{P_{2}^{2}+P_{3}^{2}}\right) \frac{d N}{d^{2} P_{T}}}{\int d^{2} P_{T} \frac{d N}{d^{2} P_{T}}},
$$

where $P^{i}$ is the momentum of the fluid in the $X^{i}$ coordinate and $d^{2} P_{T}=d P^{2} d P^{3}$. To consider $v_{2}$ in the present model, we introduce a coarse-grained (i.e., averaged over a small volume) momentum flow,

$$
P^{i}=K^{i}(x, \tau) .
$$

At each fixed time $\tau$, this can be considered as a mapping from the $P^{i}$-space to the $x^{i}$-space 6 Now $v_{2}$ can be expressed as an integral over the co-moving coordinates:

$$
v_{2}=\frac{\int d x^{2} d x^{3}\left(\frac{P_{2}^{2}-P_{3}^{2}}{P_{2}^{2}+P_{3}^{2}}\right)_{x} \rho_{N}(x)}{\int d x^{2} d x^{3} \rho_{N}(x)},
$$

where $\rho_{N}(x)=\frac{d N}{d x^{2} d x^{3}}$ is the particle density in the transverse space. Notice that the Jacobians cancel out.

To proceed, we need an explicit expression for $K(x)$. We first relate the coordinates of the Kasner spacetime and the usual Minkowski spacetime. We can identify the flat space variable $X^{i}$ for $i=2,3$ by

$$
X^{i} \simeq \tau^{a_{i}} x^{i} \text { near } x^{i}=0,
$$

which is nothing but (A.2) under (A.5). Then the fluid momentum in the flat space is given by

$$
P^{i}=\rho \frac{d X^{i}}{d \tau}=\rho a_{i} \tau^{a_{i}-1} x^{i}
$$

Here $\rho=m \rho_{N}$ is a mass density with some proper mass parameter $m$. Since we treat it as a constant from now on, it is irrelevant in the calculation below.

A few technical remarks are in order:

1. One should notice that the fluid momentum $P^{i}(x)$ is different from the individual particle momentum. By replacing the momentum by fluid momentum, we expect a small deviation from the original $v_{2}$. So one may want to call the final expression by $\bar{v}_{2}$. However, this is precisely what we should have when we describe the system by hydrodynamics where everything is to be defined in the coarse-grained level.

\footnotetext{
${ }^{6}$ In the co-moving coordinate, where the fluid is at rest, we do not have any flow. So $P^{i}(x)$ is the coarse-grained momentum field in Minkowski space written as a function of co-moving coordinate.
} 


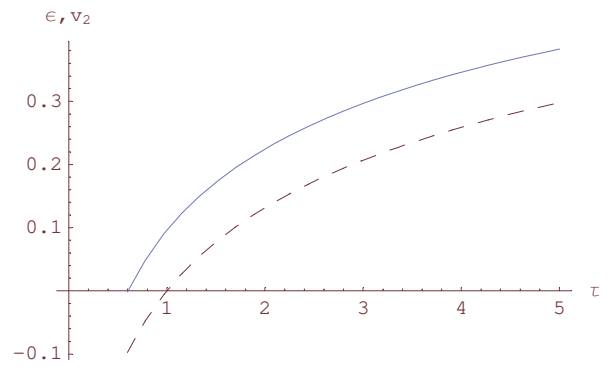

Figure 2: Time evolution of the elliptic flow parameters. Dotted line is the eccentricity and the solid line is the $v_{2}$ for $b=0.1$.

2. Since our approximation is valid in the small $x^{i}$ region, the integrals in (4.8) are now defined within the small $x^{i}$ region by introducing a cut off radius.

The resulting $v_{2}$ can be calculated in our model to yield

$$
v_{2}=\frac{b \tau^{b}+c \tau^{c}}{b \tau^{b}-c \tau^{c}}
$$

for $b>0, c<0$. Comparing this with the result for the eccentricity in the small $x^{i}$ region

$$
\varepsilon=\frac{\tau^{2 b}-\tau^{2 c}}{\tau^{2 b}+\tau^{2 c}}
$$

we plot the time evolution of $v_{2}$ in figure 2 .

Notice that the hydrodynamics describes relatively late time regime and we do not consider the time region where $v_{2}$ is negative. At the time of zero $v_{2}, \varepsilon$ is negative and its absolute value decreases to zero. That is, the fireball core becomes more round in the transverse space. After that initial period, $\varepsilon$ becomes positive and grows in the same direction of growing $v_{2}$. This is qualitatively the same behaviour as those given in [15, 16] and [20]. Notice that there is only one independent parameter $b$ that can be used to parameterize the initial eccentricity.

\section{Discussion}

In this paper, we extended the Bjorken hydrodynamics to the case of anisotropic three-dimensional expansion. The Ricci flat condition suggested by the conformal invariance imposes the condition on the anisotropy parameters. As a consequence, the four-dimensional boundary metric becomes precisely that of Kasner universe. Our hydrodynamic equation of motion is independent of the anisotropic parameters, so that it is the same as that obtained by Bjorken.

Although the Kasner spacetime is a curved spacetime, we found that it gives a well-approximated local rest frame of an anisotropically expanding fluid on the flat spacetime. We obtained the eccentricity and $v_{2}$ of the elliptic flow of the fluid. The expansion in this setting has a deviation from that of realistic RHIC fireballs, since one of the transverse direction contracts as a consequence of the 
Kasner condition. This deviation is essentially due to the conformal invariance. Whether one can lessen the condition of the conformal invariance is an important issue, which we will treat in other publication.

We also extended the "falling horizon solution" obtained in [9, 14] to the case of three-dimensionally expanding fluid. It is very important to figure out how to use the bulk metric apart from reading off the horizon location, which gives the cooling rate and the entropy creation rate. It is also interesting to workout the details of the Hawking evaporation and Wilson line calculations for the external quark-antiquark in our time-dependent metric. We will come back to these issues in later publication.

\section{Acknowledgments}

We thank T. Hirano and E. Shuryak for valuable comments on the manuscript and interesting suggestions. S.N. thanks the Yukawa Institute for Theoretical Physics at Kyoto University. Discussions during the YITP workshop YITP-W-99-99 on "thermal quantum field theories and their applications" were useful to improve the section 4. This work was supported by the SRC Program of the KOSEF through the Center for Quantum Space-time(CQUeST) of Sogang University with grant number R11 - 2005 -021 and also by KOSEF Grant R01-2004-000-10520-0.

\section{A Relationship between Kasner spacetime and our local rest frame}

We clarify the relationship between our local rest frame and the Kasner spacetime in the following strategy. We start from the cartesian coordinate and make a coordinate transformation to define our local rest frame. The transformation is essentially what we have employed at (4.9). At this stage, we are still on the flat spacetime but the local rest frame is that of the anisotropically expanding fluid with elliptic flow. Next, we see that the local rest frame is equivalent to the Kasner spacetime under a well-controled approximation which is specified at the end of this section.

Let us start from the cartesian coordinate,

$$
d s^{2}=-\left(d X^{0}\right)^{2}+\left(d X^{1}\right)^{2}+\left(d X^{2}\right)^{2}+\left(d X^{3}\right)^{2}
$$

and perform the following coordinate transformation

$$
\left(X^{0}, X^{1}, X^{2}, X^{3}\right)=\left(\tau \cosh x^{1}, \tau \sinh x^{1}, \tau^{b} x^{2}, \tau^{c} x^{3}\right)
$$

to define our local rest frame $\left(\tau, x^{1}, x^{2}, x^{3}\right)$. Notice that the $\left(X^{0}, X^{1}\right)$ part has been "boosted" with rapidity $x^{1}$ and the $\left(X^{2}, X^{3}\right)$ part has been transformed by (4.9). The resulting metric on the local rest frame is given by

$$
d s^{2}=-d \tau^{2}+\tau^{2 a}\left(\tau^{2-2 a}\right)\left(d x^{1}\right)^{2}+\tau^{2 b}\left(d x^{2}+b \frac{x^{2}}{\tau} d \tau\right)^{2}+\tau^{2 c}\left(d x^{3}+c \frac{x^{3}}{\tau} d \tau\right)^{2} .
$$

The parameters $a, b, c$ are free so far. 
Next, let us impose the Kasner condition (2.3) among $a, b, c$ and assume $b \ll 1 . a$ and $c$ is then given by $a=1-3 b^{2}$ and $c=-b+3 b^{2}$ approximately. Now (A.3) is written as

$$
\begin{aligned}
d s^{2}= & -d \tau^{2}+\tau^{2 a}\left(1+6 b^{2} \log \tau+\cdots\right)\left(d x^{1}\right)^{2} \\
& +\tau^{2 b}\left(d x^{2}+b \frac{x^{2}}{\tau} d \tau\right)^{2}+\tau^{2 c}\left(d x^{3}+\left(-b+3 b^{2}+\cdots\right) \frac{x^{3}}{\tau} d \tau\right)^{2} .
\end{aligned}
$$

The above metric agrees with the Kasner metric (2.2) under the following approximation:

$$
\frac{\left|x^{2}\right|}{\tau} \sim \frac{\left|x^{3}\right|}{\tau} \sim b \ll 1, \text { with } b \log \tau \sim O(1) .
$$

We ignore $O\left(b^{2}\right)$ contributions while we $\operatorname{keep} b \log \tau$. This is a mixture of small $b$ approximation (we consider almost central collision), small $\left(x^{2}, x^{3}\right)$ approximation (we consider only within the central part of the QGP) and the late time approximation. The condition $b \log \tau \sim O(1)$ is necessary for us to get the nontrivial results.

\section{References}

[1] J. M. Maldacena, "The Large N Limit of Superconformal Field Theories and Supergravity," Adv. Theor. Math. Phys. 2 (1998) 231, Int. J. Theor. Phys. 38 (1999) 1113, hep-th/9711200;

S. S. Gubser, I. R. Klebanov and A. M. Polyakov, "Gauge Theory Correlators from Non-Critical String Theory," Phys. Lett. B428 (1998) 105, hep-th/9802109;

E. Witten, "Anti De Sitter Space and Holography," Adv. Theor. Math. Phys. 2 (1998) 253, hep-th/9802150.

[2] S. J. Sin and I. Zahed, "Holography of radiation and jet quenching," Phys. Lett. B608 (2005) 265, hep-th/0407215.

[3] H. Nastase, "The RHIC fireball as a dual black hole," hep-th/0501068;

H. Nastase, "DBI skyrmion, high energy (large s) scattering and fireball production," hep-th/0512171;

H. Nastase, "More on the RHIC fireball and dual black holes," hep-th/0603176.

[4] E. Shuryak, S-J. Sin and I. Zahed, "A Gravity Dual of RHIC Collisions," hep-th/0511199.

[5] O. Aharony, S. Minwalla and Toby Wiseman, "Plasma-balls in large N gauge theories and localized black holes," Class. Quant. Grav. 23 (2006) 2171, hep-th/0507219.

[6] E. V. Shuryak, "What RHIC Experiments and Theory tell us about Properties of Quark-Gluon Plasma?" Nucl. Phys. A750 (2005) 64, hep-ph/0405066.

[7] M. J. Tannenbaum, "Recent results in relativistic heavy ion collisions: from "a new state of matter" to "the perfect fluid"," Rept. Prog. Phys. 69 (2006) 2005, nucl-ex/0603003. 
[8] For earlier attempt for QCD-like models, see for example following reviews and references therein: O. Aharony, "The non-AdS/non-CFT correspondence, or three different paths to QCD," hep-th/0212193;

A. Zaffaroni, "RTN lectures on the non AdS/non CFT Correspondence," PoS(RTN2005)005 at http://pos.sissa.it/.

More recent proposals are :

T. Sakai and S. Sugimoto, "Low energy hadron physics in holographic QCD," Prog. Theor. Phys. 113 (2005) 843, hep-th/0412141;

T. Sakai and S. Sugimoto, "More on a holographic dual of QCD," Prog. Theor. Phys. 114 (2006) 1083, hep-th/0507073;

J. Erlich, E. Katz, D. T. Son and M. A. Stephanov, "QCD and a holographic model of hadrons," Phys. Rev. Lett. 95 (2005) 261602, hep-ph/0501128;

L. Da Rold and A. Pomarol, "Chiral symmetry breaking from five dimensional spaces," Nucl. Phys. B721 (2005) 79, hep-ph/0501218.

[9] R. A. Janik and R. Peschanski, "Asymptotic perfect fluid dynamics as a consequence of AdS/CFT," Phys. Rev. D73 (2006) 045013, hep-th/0512162.

[10] R. A. Janik and R. Peschanski, "Gauge/gravity duality and thermalization of a boost-invariant perfect fluid," Phys. Rev. D74 (2006) 046007, hep-th/0606149.

[11] S. de Haro, K. Skenderis and S. N. Solodukhin, "Holographic Reconstruction of Spacetime and Renormalization in the AdS/CFT Correspondence," Commun. Math. Phys. 217 (2001) 595, hep-th/0002230.

[12] K. Skenderis, "Lecture Notes on Holographic Renormalization," Class. Quant. Grav. 19 (2002) 5849, hep-th/0209067.

[13] J. D. Bjorken, "Highly relativistic nucleus-nucleus collisions: The central rapidity region," Phys. Rev. D27 (1983) 140.

[14] S. Nakamura and S-J. Sin, "A holographic dual of hydrodynamics," JHEP 09 (2006) 020, hep-th/0607123.

[15] P. F. Kolb and U. Heinz, "Hydrodynamic description of ultrarelativistic heavy-ion collisions," nucl-th/0305084.

[16] E. Shuryak, "Why does the Guark Gluon Plasma at RHIC behave as a nearly ideal fluid?" Prog. Part. Nucl. Phys. 53 (2004) 273, hep-ph/0312227.

[17] D. Teaney, J. Lauret and E. V. Shuryak, "Flow at the SPS and RHIC as a quark gluon plasma signature," Phys. Rev. Lett. 864783 (2001), nucl-th/0011058.

[18] P. F. Kolb, P. Huovinen, U. W. Heinz and H. Heiselberg, "Elliptic flow at SPS and RHIC: From kinetic transport to hydrodynamics," Phys. Lett. B500 232 (2001), hep-ph/0012137. 
[19] T. Hirano, U. W. Heinz, D. Kharzeev, R. Lacey and Y. Nara, "Hadronic dissipative effects on elliptic flow in ultrarelativistic heavy-ion collisions," Phys. Lett. B636 299 (2006), nucl-th/0511046.

[20] B. Zhang, M. Gyulassy and C. M. Ko, "Elliptic flow from a parton cascade," Phys. Lett. B455 (1999) 45, nucl-th/9902016.

[21] G. Policastro, D. T. Son and A. O. Starinets, "Shear viscosity of strongly coupled N=4 supersymmetric Yang-Mills plasma," Phys. Rev. Lett. 87 (2001) 081601, hep-th/0104066.

[22] T. Hirano and M. Gyulassy, "Perfect Fluidity of the Quark Gluon Plasma Core as Seen through its Dissipative Hadronic Corona," Nucl. Phys. A769 (2006) 71, nucl-th/0506049.

[23] S. Nakamura and S-J. Sin, work in progress. 